\section{Development of diffuse large B-cell lymphoma in a patient with Waldenström's macroglobulinemia/ lymphoplasmacytic lymphoma: clonal identity between two B-cell neoplasms}

\author{
Masayuki Shiseki, ${ }^{1}$ Akihiro Masuda, ${ }^{2}$ \\ Norimichi Watanabe, ${ }^{3}$ Masami Fujii, ${ }^{3}$ \\ Tomofumi Kimura, ${ }^{4}$ Kentaro Yoshinaga, ${ }^{1}$ \\ Naoki Mori, ${ }^{1}$ Masanao Teramura, ${ }^{1}$ \\ Toshiko Motoji ${ }^{1}$ \\ ${ }^{1}$ Department of Hematology; \\ 2Department of Surgical Pathology, \\ Tokyo Women's Medical University, \\ Tokyo; ${ }^{3}$ Division of Hematology; \\ ${ }^{4}$ Division of Hematology/oncology and \\ Transfusion Medicine, KKR Suifu \\ Hospital, Ibaraki, Japan
}

\begin{abstract}
Waldenström's macroglobulinemia (WM)/ lymphoplasmacytic lymphoma (LPL) is an indolent mature B-cell neoplasm. In rare cases of WM/LPL, diffuse large B-cell lymphoma (DLBCL) develops as a result of histologic transformation. In this report, we present a case of DLBCL developing in a patient with WM/LPL. Combination chemotherapy for DLBCL was effective and complete remission was eventually achieved. We attempted to determine the clonal relatedness between WM/LPL and DLBCL in the patient by analyzing complementarity-determining region 3 (CDR3) in the immunoglobulin heavy chain gene. A common CDR3 sequence was found in tumor cells of DLBCL and those of WM/LPL, indicating that tumor cells of DLBCL are clonally identical to those of WM/LPL. Therefore, in the present case, DLBCL is developed from WM/LPL cells by clonal evolution.
\end{abstract}

\section{Introduction}

Waldenström's macroglobulinemia (WM), a rare B-cell neoplasm characterized by lymphoplasmacytic cell proliferation in bone marrow with IgM monoclonal gammopathy, is classified as a subtype of lymphoplasmacytic lymphoma (LPL) in WHO classification. ${ }^{1-3}$ Although WM/LPL is an indolent disorder and the median overall survival of patients with WM/LPL is in the range of 5-10 years, diffuse large B-cell lymphoma (DLBCL) develops as a result of histologic transformation in approxi- mately $5-10 \%$ of patients with WM/LPL. ${ }^{4-6}$ Once DLBCL occurs in patients with WM/LPL, the disease course turns more aggressive. In general, DLBCL developing in a patient with WM/LPL is resistant to chemotherapy and the prognosis is poorer than that of de novo DLBCL. Median survival from the development of DLBCL is only 2-5 months even when combination chemotherapy for aggressive lymphoma is applied. ${ }^{6}$

It has been debated whether DLBCL cooccuring with WM/LPL develops by clonal evolution or as a secondary malignancy. To address this issue, the clonal relatedness between tumor cells of WM/LPL and those of DLBCL have been investigated by different procedures. Now, the clonality of mature B-cell neoplasm can be determined more precisely by analyzing complementarity determining region 3 (CDR3) in the immunoglobulin heavy chain $(I G H)$ gene by a PCR-based molecular biological technique. ${ }^{7}$ To date, three groups have independently reported on the clonal relatedness between tumor cells of WM/LPL and those of co-occurring DLBCL by analyzing CDR3 sequences..$^{8-10}$ One group showed clonal identity between tumor cells of WM/LPL and those of co-occuring DLBCL, ${ }^{8}$ while two other groups showed that tumor cells of DLBCL are not clonally identical to those of WM/LPL tumor cells. ${ }^{9,10}$ In this report, we present a case of DLBCL developing in a patient with WM/LPL. We attempted to determine clonal relatedness between tumor cells of WM/LPL and those of DLBCL by analyzing CDR3 sequences.

\section{Case Report}

A 63-year-old man was diagnosed with WM/LPL based on findings including increased serum IgM $(4823 \mathrm{mg} / \mathrm{dL})$ with a monoclonal component and lymphoplasmacytic cell proliferation in bone marrow. He received chemotherapy consisted of ranimustine, vindesine, melphalan, and prednisolone. After completion of the first cycle of therapy, serum IgM level was decreased to $3058 \mathrm{mg} / \mathrm{dL}$. Three years after diagnosis of WM/LPL, supraclavicular lymph node swelling was found on physical examination, and an abnormal nodular lesion in lung field was observed on chest X-ray. Supraclavicular lymph node biopsy was performed for pathological diagnosis. Histopathological studies on supraclavicular lymph node revealed diffuse proliferation of large B-cells positive for CD20, CD79a and lambda chains by immunohistochemistry, leading to a pathological diagnosis of DLBCL (Figure 1A and B). Chemotherapy by a THP-COP regimen consisting of cyclophosphamide, epirubicin, vincristine, and prednisolone was initiated.
Correspondence: Masayuki Shiseki, 8-1, Kawadacho, Shinjuku-ku, Tokyo 162-8666, Japan. Tel: +81.3-3353.8111 ext.31544 -

Fax: +81.3.5269.7329.

E-mail: shisekim@dh.twmu.ac.jp

Key words: Waldenström's macroglobulinemia, lymphoplasmacytic lymphoma, diffuse large Bcell lymphoma, complementarity determining region 3 .

Contributions: MS, clinical data collection and analysis, clonarity analysis of tumor cells including PCR, subcloning and sequencing, and manuscript preparation; AM, pathological diagnosis, preparation of figures for manuscript; NW, MF, TK, clinical data collection and analysis; KY, NM, partially involved in clonarity analysis of tumor cells, including DNA extraction from samples and electrophoresis of PCR products; MT, support for clinical and experimental data analysis. TM, organizing and supervising the present study.

Conflict of interest: the authors report no conflicts of interest.

Received for publication: 28 May 2011.

Revision received: 25 July 2011.

Accepted for publication: 26 July 2011

This work is licensed under a Creative Commons Attribution NonCommercial 3.0 License (CC BYNC 3.0).

(C) Copyright M. Shiseki, et al., 2011

Licensee PAGEPress, Italy

Hematology Reports 2011; 3:e10

doi:10.4081/hr.2011.e10

Treatment was effective and lung tumor disappeared and lymph node swelling was improved. Subsequently, complete remission was achieved after the completion of six cycles of THP-COP therapy. Five years after completion of THP-COP therapy, when he was the age of 71-year-old, disease status was re-evaluated. There was no sign of relapse of DLBCL, while serum IgM level was still high, and bone marrow showed proliferation of lymphoplasmacytic cells $(11.7 \%$ of all nucleated cells) positive for CD79a, cytoplasmic IgM, and lambda chain but not for CD20, without large B-cell proliferation (Figure 1C and D)

\section{Materials and Methods}

\section{Samples and DNA extraction}

Written informed consent regarding genetic analysis of tumor cells was obtained from the patient. Mononuclear cells were separated from the bone marrow sample as described previously. ${ }^{11}$ Genomic DNA was extracted from bone marrow mononuclear cells using QIAamp 
DNA Blood Mini kit (QIAGEN Sciences, Hilden, Germany) and from the paraffin-embedded lymph node sample by using DEPAX kit (TAKARA BIO., Ohtsu, Shiga, Japan) according to the manufacturers' instructions.

\section{Polymerase chain reaction}

Semi-nested polymerase chain reaction (PCR) was carried out to amplify CDR3 sequences in the $I G H$ gene as described previously with minor modifications, using primer sets Fr3A (5'-ACACGGC [C/T] [G/C]TGTATTACTGT -3') and LJH (5'-TGAGGAGACGGTGACC-3') for first round amplification, and Fr3A and VLJH (5'GTGACCAGGGT[A/G/C/T] CCTTGGCCCCAG3') for second round amplification.7 First round PCR was carried out using $100 \mathrm{ng}$ of genomic DNA as a template in $50 \mu \mathrm{L}$ of a reaction mixture containing $2 \mu \mathrm{L}$ of $10 \mathrm{X}$ buffer, $1.5 \mathrm{mM} \mathrm{MgCl} 2,250 \mathrm{nM}$ of each deoxynucleotide, 0.2 units of rTaq DNA polymerase (TAKARA BIO.), and $300 \mathrm{nM}$ of each primer. Forty cycles of $94^{\circ} \mathrm{C}$ for $30 \mathrm{~s}, 57^{\circ} \mathrm{C}$ for $30 \mathrm{~s}$, and $72^{\circ} \mathrm{C}$ for $60 \mathrm{~s}$ were performed. Second round PCR was carried out using purified first round PCR product as a template in $50 \mu \mathrm{L}$ of the same reaction mixture as that for first round PCR except for the primer sets. Forty cycles of $94^{\circ} \mathrm{C}$ for $30 \mathrm{~s}, 60^{\circ} \mathrm{C}$ for $30 \mathrm{~s}$, and $72^{\circ} \mathrm{C}$ for $60 \mathrm{~s}$ were performed.

\section{Subcloning of polymerase chain reaction products and DNA sequencing}

Sequences of PCR products of CDR3 were determined after subcloning as described previously. ${ }^{11}$ Briefly, PCR products were purified with the QIAquick PCR purification kit (QIAGEN Sciences), and cloned into pGEM-T vector (Promega, Madison, WI, USA). After bacterial transformation, plasmids were purified and subjected to sequence determination. Both strands of each PCR product were sequenced using DYEnamic ET Dye Terminator sequencing kit (Amersham, Buckinghamshire, UK) and the MegaBase sequence system (Amersham) according to the manufacturer's instructions.

\section{Results}

We analyzed sequences of CDR3 by PCR from the lymph node biopsy specimen, by which the diagnosis of DLBCL was made, and from the bone marrow sample, in which lymphoplasmacytic cell proliferation was observed. PCR products were cloned into pGEM-T vector, and then nucleotide sequences of PCR products were determined after subcloning. For lymph the node specimen, eight bacterial colonies were isolated and subjected to sequence analysis. Six of the eight bacterial colonies contained the same nucleotide sequence (Figure 2), and the remaining two did not contain any CDR3 sequence. For the bone marrow specimen, 16 bacterial colonies were isolated and subjected to sequence analysis. Six out of the 16 bacterial colonies did not contain any CDR3 sequences. The remaining ten bacterial colonies contained seven different CDR3 sequences (A-G), and three colonies contained " $\mathrm{A}$ " sequence, which is identical to the CDR3 sequence identified in lymph node (Table 1). Subsequently, we found a common CDR3 sequence in DLBCL cells (lymph node) and WM/LPL cells (bone marrow).

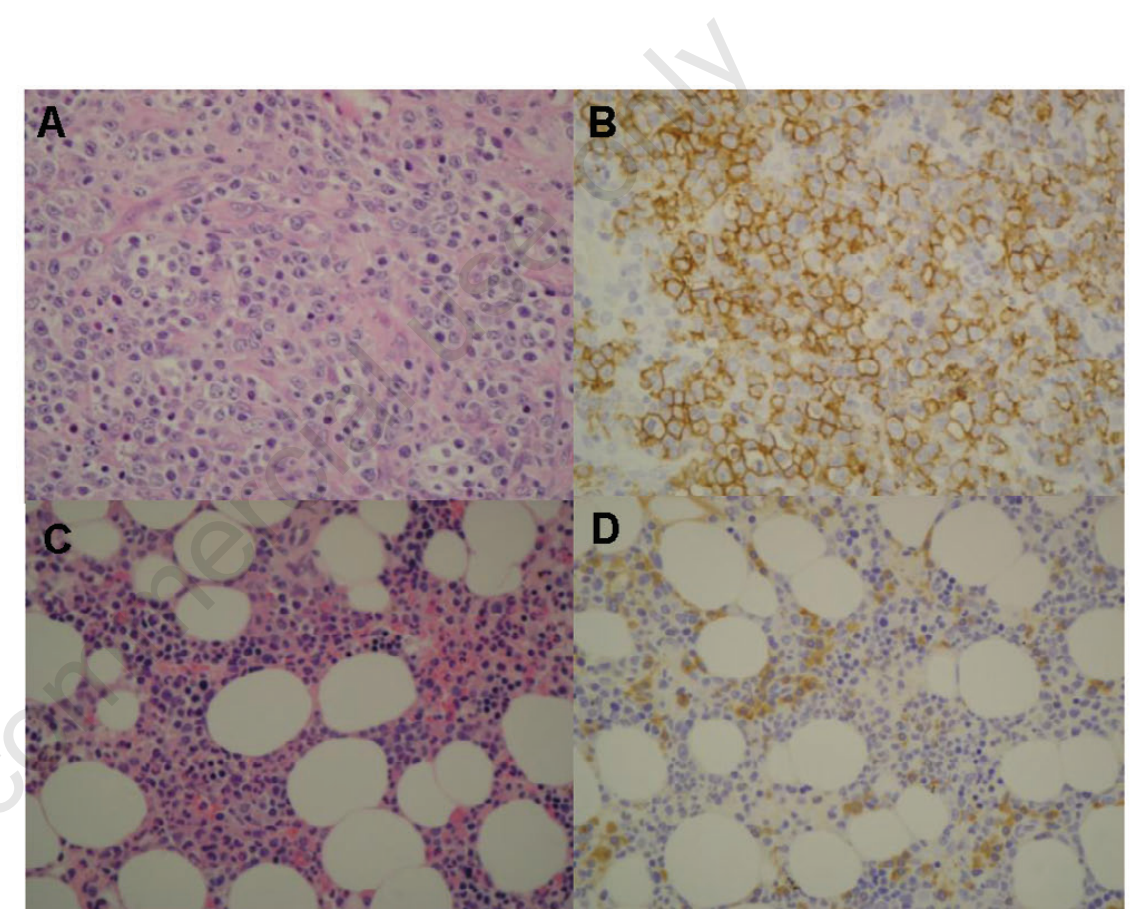

Figure 1. Histopathology of lymph node and bone marrow tissues. A) In lymph node, proliferation of large tumor cells with diffuse pattern is observed (HE staining X400); B) and tumor cells are positive for CD20 (Immunohistochemistry, X400). C) In bone marrow, proliferation of lymphoplasmacytic cells is observed (HE staining, X400); D) and these cells are positive for CD79a (Immunohistochemistry, X400).

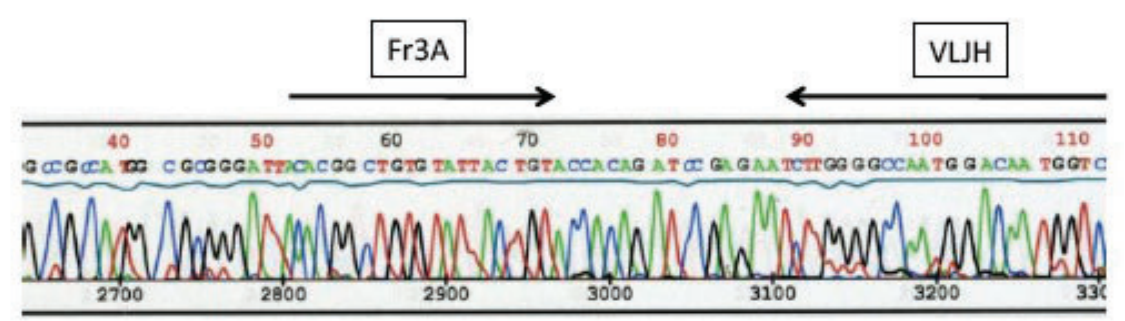

Figure 2. Sequence analysis of CDR3 in lymph node. Arrows indicate primer sequences of Fr3A and VLJH.

\section{Discussion}

The results of analysis of the CDR3 sequence in the $I G H$ gene shows clonal identity between tumor cells of WM/LPL and those of co-occurring DLBCL, indicating that DLBCL are originated from WM/LPL by clonal evolution in the present case. It has been debated whether tumor cells of co-occurring DLBCL are identical to those of WM/LPL. To date, the clonal relatedness between tumor cells of WM/LPL and those of co-occurring DLBCL by analyzing CDR3. Similar to the present result, Nakamura et al. showed clonal identity between tumor cells of WM/LPL and those of Shimizu et al. and Tojo et al. showed clonal dif- 
ference between tumor cells of DLBCL and those of WM/LPL, suggesting that DLBCL develops independently as a second neoplasm in patients with WM/LPL. ${ }^{9,10}$ Collectively, these discordant results suggest that there are two different pathways of development of DLBCL in patients with WM/LPL: clonal evolution or the development of a second neoplasm. Histologic transformation occurs in other types of indolent B-cell neoplasms, including chronic lymphocytic leukemia (CLL). Clonal relatedness between tumor cells of co-occuring DLBCL and those of CLL has been investigated more extensively, and the results indicate that DLBCL can develop either by clonal evolution or as a secondary neoplasm. ${ }^{12,13}$ In the majority of patients with CLL, DLBCL occurs by clonal evolution, suggesting that clonal evolution is a major pathway of the development of DLBCL in cases of CLL. ${ }^{12,13}$ Similar to CLL, in cases of WM/LPL, DLBCL develops by these two pathways, although it is not clear which of two pathways is dominant in the development of DLBCL in WM/LPL.

The molecular mechanism of the development of DLBCL by clonal evolution in WM/LPL has not been elucidated. In the case of CLL, several different events, including additional genetic alterations, and viral infection, may trigger clonal evolution. ${ }^{13-16}$ For instance, the acquisition of $p 53$ tumor suppressor gene mutation and/or chromosome 17p13 deletion, and c-MYC alterations frequently occur in tumor cells during histologic transformation to DLBCL. ${ }^{13,14,17}$ Epstein-Barr virus infection has been identified in lymphoma cells in a few patients with co-occurring aggressive lymphoma with CLL. ${ }^{18}$ Similar events may occur during clonal evolution in DLBCL development in patients with WM/LPL. Further investigation is necessary.

It is also important to clarify whether there are any differences in biological behaviors of tumor cells and clinical features between DLBCL developed by clonal evolution and that developed as a secondary neoplasm. Once DLBCL develops in WM/LPL patients, the disease course turns more aggressive. In general, the response to chemotherapy is not good and median overall survival is only 3-5 months, even when multi-agent chemotherapy is applied. ${ }^{6}$ The introduction of rituximab to chemotherapy may improve the treatment outcome of DLBCL developed in WM/LPL patients. ${ }^{19,20}$ When the patient in the present case received therapy for DLBCL, rituximab had not been available in Japan. Combination chemotherapy (THP-COP therapy) without rituximab was, however, effective for DLBCL, and the patient eventually achieved complete remission and maintained it for approximately five years. Interestingly, as described in report by Nakamura et al., a patient with co-occurring

Table 1. Summary of CDR3 sequences observed in bone marrow.

CDR3 sequences

Number *

of subclones

A $\quad 5^{\prime}$-(Fr3A)-ACCACAGATCCGAGAAT-(VUH)-3'

B 5'-(Fr3A)-GCGAGACAACGCGAGTCATTGTAGTGTACCAGCTGCTATACGT 1 CGCTGCTTTTGATTTTGATAT-(VUH)-3'

C 5'-(Fr3A)-GCGAGACAACGCGAGTCATTGTAGTAGTACCAGCTATACGTCG 1 GTGCTTTTGATAT-(VUH)-3'

D 5'-(Fr3A)-TGTCGAAAAGCTTCATTAGTATGGTTCGGGGAGATTGACTA-(VLJH)-3' 1

E 5'-(Fr3A)-GCGAGTACGTATTACGATTTTTGGAGTGGTTATTATCGGAAATT 1 GACTA-(VDH)-3'

F 5'-(Fr3A)-ACCACCCAAGGGCCCCCACCCCCGGATATTTTGACTGGTTATTA 2 CCCCTTATCATTTGACTA-(VLH)-3'

G 5'-(Fr3A)-GCAAAAGCTTCATTACTATGGTTCGGGGAGATTGACTA-(VLJH)-3'

1

* Subclones that did not contain any CDR3 sequences are excluded.

DLBCL caused by tumor cells clonally identical to WM/LPL achieved complete remission by combination chemotherapy. ${ }^{8}$ In contrast, as described in reports by Shimizu et al. and Tojo et al., patients with DLBCL caused by different clones from those of WM/LPL showed poorer prognosis, and died within several months. ${ }^{9,10}$ However, the number of analyzed cases is too small to clarify clinical implication of clonal relatedness. Currently, Rossi et al. performed comprehensive molecular study including analysis of clonal relatedness of tumor cells of CLL and co-occurring DLBCL, indicating that clonally unrelated cases are clinically and biologically distinct from clonally related cases, and are characterized by better clinical outcomes. ${ }^{13}$ It is not clear whether clinical significance of clonal relatedness between tumor cells of co-occurring DLBCL and original tumor cells in WM/LPL is different from that in CLL. It is necessary to accumulate cases of DLBCL cooccurring with WM/LPL and to analyze correlation between clinicopathological features and clonal relatedness.

\section{References}

1. Waldenström J. Incipient myelomatosis or rssential hyperglobulinemia with fibrinogenemia-A new syndrome. Acta Med Scand 1944;117:216-22.

2. Owen RG, Treon SP, Al-Katib A, et al. Clinicopathological definition of Waldenström's macroglobulinemia: consensus panel recommendations from the Second International Workshop on
Waldenström's macroglobulinemia. Semin Oncol 2003;30:110-5.

3. Dimopoulos MA, Kyle RA, Anagnostopoulos A, Treon SP. Diagnosis and management of Waldenström's macroglobulinemia. J Clin Oncol 2005;23: 1564-77.

4. Swerdlow SH, Berger F, Pileri SA, et al. Lymphoplasmacytic lymphoma. In: Swerdlow SH, Campo E, Harris NL, Jaffe ES, Pileri SA, Stein H, Thiele J, Vardiman JW, editors. WHO classification of tumours of hematopoietic and lymphoid tissues. Lyon: IARC press; 2008. p.194-195.

5. Kyrtsonis MC, Vassilakopoulos TP, Angelopoulos MK, et al. Waldenström's macroglobulinemia: clinical course and prognostic factors in 60 patients; experience from a single hematology unit. Ann Hematol 2001;80:722-27.

6. Lin P, Mansoor A, Buenos-Ramos C, et al. Diffuse large B-cell lymphoma occurring in patients with lymphoplasmacytic lymphoma/Waldenström's macroglobulinemia: Clinicopathologic features of 12 cases. Am J Clin Pathol 2003;120:246-53.

7. Trainor KJ, Brisco MJ, Story CJ, Morley AA. Monoclonality in B-lymphoproliferative disorders detected at the DNA level. Blood 1990;75:2220-2.

8. Nakamura N, Kuze T, Hashimoto Y, et al. Analysis of the immunoglobulin heavy chain gene of secondary diffuse large Bcell lymphoma that subsequently developed in four cases with B-cell chronic lymphocytic leukemia or lymphoplasmacytoid lymphoma (Richter syndrome). Pathol Int 2000;50:636-43. 
9. Shimizu S, Tomonaga Y, Kojima H, et al. Simultaneous development of lymphoplasmacytic lymphoma and diffuse B-cell lymphoma - analyses of the clonal relatedness by sequencing CDR3 in immunoglobulin heavy chain genes. Eur J Haematol 2003;70:119-24.

10. Tojo K, Hattori T, Ito T, et al. Multiple brain tumors of diffuse large B-cell lymphoma in a patient with Waldenström's macroglobulinemia/lymphoplasmacytic lymphoma: PCR and DNA sequence analysis show evidence of differences in clonality of the two B-cell malignancies. Internal Med 2004;43: 990-6.

11. Shiseki M, Kitagawa Y, Wang Y-H, et al. Lack of nucleophosmin mutation in patients with myelodysplastic syndrome and acute myeloid leukemia with chromosome 5 abonormalities. Leukemia
Lymphoma 2007;48:2141-4.

12. Mao Z, Quintanilla-Martinez L, Raffeld M, et al. IgVH mutational status and clonality analysis of Richter's transformation. Am J Surg Pathol 2007;31:1605-14.

13. Rossi D, Valeria V, Deambrogi C, et al. The genetics of Richter syndrome reveals disease heterogeneity and predicts survival after transformation. Blood 2011:117:3391401.

14. Rossi D, Cerri M, Capello D, et al. Biological and clinical risk factors of chronic lymphocytic leukemia transformation to Richter syndrome. Br J Haematol 2008:142:202-15.

15. Nakamura N, Abe M. Richter syndrome in B-cell chronic lymphocytic leukemia. Pathol Int 2003;53:195-203.

16. Tsimberidou AM, Keating MJ. Richter Syndrome. Cancer 2005;103:216-28.
17. Fenaux P, Preudohomme C, Lai JL, et al. Mutations of the p53 gene in B-cell chronic lymphocytic leukemia: a report on 39 cases with cytogenetic analysis. Leukemia 1992;6:246-50.

18. Ansell SM, Li CY, Lloyd RV, Phyliky RL. Epstein-Barr virus infection in Richter's transformation. Am J Hematol 1999;60:99104.

19. Dimopoulos M, Zervas C, Zomas A, et al. Treatment of Waldenström's macroglobulinemia with rituximab. J Clin Oncol 2002;20:2327-33.

20. Uchino K, Sameshima H, Miyamoto T, et al. Successful treatment of diffuse large Bcell lymphoma following Waldenström's macroglobulinemia with CHOP chemotherapy followed by combination therapy of CHOP with rituximab. Internal Medicine 2004;43:131-4. 